\title{
Heide-Marie Lauterer
}

\section{„Ja, das haben wir miterlebt, aber wir konnten dagegen ja nichts machen"}

\author{
Diakonische Werke und Verbände \\ in der Konsolidierungsphase des NS-Gewaltregimes \\ am Beispiel des Kaiserswerther Verbandes
}

Der 1916 gegründete „Kaiserswerther Verband deutscher Diakonissenmutterhäuser", auf den ich mich im folgenden im wesentlichen beziehen werde', war 1933 der größte evangelische Schwesternverband im Bereich der Inneren Mission. Ihm gehörten ca. 28000 Diakonissen und 3700 diakonische Hilfskräfte in 69 über das ganze Reich verteilten Mutterhäusern an. Die Diakonissen arbeiteten im Erziehungswesen, in der geschlossenen und offenen Fürsorgearbeit, in Anstalten für körperlich und geistig Behinderte sowie im Pflegebereich als Gemeindeund Krankenschwestern. Gemäß der Verbandssatzung, den sog. Grundordnungen, geschah das diakonische Handeln im Auftrag Jesu Christi. Im Vergleich zum humanitär begründeten Dienst des Deutschen Roten Kreuzes und dem politischen Dienst der NS-Schwesternschaft war der Diakonissendienst als religiös motivierte Liebestätigkeit an „Armen und Kranken “ definiert ${ }^{2}$. Der Kaiserswerther Verband war dem 1848 gegründeten Centralausschuß für die Innere Mission angeschlossen.

Die einzelnen Mutterhäuser waren nach dem patriarchalischen Familienmodell organisiert. Den Hausvorstand bildeten der ordinierte Pastor als Vorsteher und neben ihm - gemäß dem biblischen Grundsatz: Der Mann ist des Weibes Haupt die Oberin als Vorsteherin. Die Oberin war selbst Diakonisse, unverheiratet und Teil der Schwesterngemeinschaft. Als Mitglied des Hausvorstandes war sie gleichzeitig „Hausmutter" und nächste Vorgesetzte der Schwestern. Die Diakonissen nahmen in diesem Gefüge die Stellung von Töchtern ein. Als Schwestern lebten

1 Vgl. Heide-Marie Lauterer, Liebestätigkeit für die Volksgemeinschaft. Der Kaiserswerther Verband Deutscher Diakonissenmutterhäuser in den ersten Jahren des NS-Regimes (Göttingen 1994). Hier finden sich auch weiterführende Literatur und Quellenangaben, auf die ich im folgenden weitgehend verzichten werde. Im folgenden zitiert: Lauterer, Liebestätigkeit. 2 „Der Armen- und Krankenfreund“, so lautet der Titel einer Zeitschrift, die Theodor Fliedner, der Begründer der Kaiserswerther Mutterhausdiakonie, seit 1849 herausgab. 
sie zusammen in der religiös begründeten Lebens- und Dienstgemeinschaft des Mutterhauses. Sie waren dem Hausvorstand gegenüber zum Gehorsam verpflichtet und gelobten Ehelosigkeit. Darüber hinaus unterlagen sie dem „Sendungsprinzip“, d.h. der Hausvorstand - der Vorsteher in Absprache mit der Oberin bestimmte über die Art der Ausbildung, über ihren Arbeitseinsatz, über den Ort ihrer Arbeitsstelle, über Arbeitszeit und Freizeit. Der Vorsteher schloß für die einzelnen Diakonissen Arbeitsverträge mit ihren verschiedenen Arbeitsstationen $a b$, zog ihr Gehalt ein und teilte ihnen ein Taschengeld zu. Diese Regelung galt auch für die Oberin, nicht jedoch für den Vorsteher, der als verheirateter evangelischer Pastor mit seinem Gehalt seine Familie ernährte.

Für die Kommunen und Gemeinden garantierte die Organisationsform der Kaiserswerther Mutterhausdiakonie Effektivität und Kontinuität einer professionalisierten Liebestätigkeit. Die Kaiserswerther Schwesterntracht wurde zum Markenzeichen qualifizierter Krankenpflege. Der Kaiserswerther Verband Deutscher Diakonissenmutterhäuser gehörte zu den Spitzenverbänden der freien Wohlfahrtspllege.

Ich habe in meiner 1994 erschienenen Dissertation ${ }^{3}$ ausführlich dargelegt, wie sich dieser christliche Verband unter dem Gleichschaltungsdruck von Reichsinnenministerium und NSDAP im Jahre 1933 verhielt. Ich habe die Frage zu beantworten versucht, wie es zu beurteilen sei, daß der Verbandsvorstand in den Jahren 1933-1938 vor allem versuchte, den Verband als Institution von nationalsozialistischer Einflußnahme frei zu halten und gleichzeitig den Bestand seiner Werke zu wahren. Das Ergebnis war ein doppeltes: Weil sich der Kaiserswerther Verbandsvorstand einerseits so hartnäckig für seine Bestandssicherung einsetzte und nicht nur Sachwerte, sondern auch die religiös begründete, traditionelle Arbeitsweise der Mutterhausdiakonie zu erhalten suchte, wurde er kein gleichgeschalteter oder gar nationalsozialistischer Schwesternverband. Andererseits habe ich in kritischer Auseinandersetzung mit den diakoniegeschichtlichen Arbeiten Jochen-Christoph Kaisers ${ }^{4}$ und Kurt Nowaks gezeigt, daß dieses Bemühen deshalb keineswegs als „Resistenz" zu charakterisieren ist. Mit Martin Broszat wird Resistenz verstanden als „aktives Gegenhandeln von Einzelnen oder Gruppen, ziviler Ungehorsam oder auch bloß als innere Bewahrung der dem National-

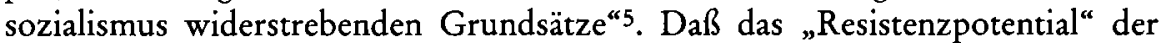
Diakonie eher einer Wunschvorstellung entsprach und dem von Kaiser geltend gemachten „zähe[n], unerbittliche[n] Kampf gegen die Euthanasie "6, so er denn stattfand, kein sichtbarer Erfolg beschieden war, zeigt die Opferstatistik. Aus den

3 Vgl. Lauterer, Liebestätigkeit 49-83.

4 Jochen-Christoph Kaiser, Sozialer Protestantismus im 20. Jahrhundert. Beiträge zur Geschichte der Inneren Mission 1914-1945 (München 1989), im folgenden zitiert: Kaiser, Protestantismus; Kurt Nowak, Euthanasie und Sterilisierung im Dritten Reich (Göttingen 1984). 5 Martin Broszat, Resistenz und Widerstand. Eine Zwischenbilanz des Forschungsprojektes Widerstand und Verfolgung in Bayern 1933-1945, in: Bayern in der NS-Zeit, Bd. IV, hrsg. von Martin Broszat, Elke Fröblich, Anton Grossmann (München 1981) 691-709.

${ }^{6}$ Kaiser, Protestantismus. 
Anstalten des Kaiserswerther Verbandes, d.h. ohne die Opfer in den Anstalten anderer Verbände der Inneren Mission hinzuzuzählen, wurden in den Jahren 1940/41 im Rahmen der „T 4 “ Aktion eine hohe Zahl an Patienten und Patientinnen in staatliche Anstalten verlegt und dort ermordet. Meine Untersuchungen haben eine Zahl von 1741 Menschen ergeben, die den Tötungsaktionen zum Opfer fielen? ${ }^{7}$. Es ist jedoch davon auszugehen, daß diese Zahl nur die Spitze des Eisbergs benennt, da der Verband über weitere, von mir nicht untersuchte Häuser verfügte, die geistig und körperlich behinderte Menschen pflegten.

Während ich in meiner Dissertation vor allem das Verhalten, das organisatorische Handeln sowie die politische und kirchenpolitische Ideologie des Verbandsvorstandes, der sich bis auf eine Ausnahme aus Männern zusammensetzte, untersucht habe, so möchte ich heute danach fragen, wie die Diakonissenschaft, d.h. die weibliche Verbandsbasis, zu dem neuen Regime stand. Mich interessiert, ob und wie sich die gewaltsame politische Umstrukturierung der Gesellschaft, die Gleichschaltung der Verbände, die Ausschaltung der Parteien, das Gewaltpotential, verstärkt durch die Aufhebung der Menschenrechte, durch Antisemitismus und Rassismus, auf die Einstellung und das Verhalten in der Schwesterngemeinschaft auswirkte.

Um diese Fragen zu beantworten, habe ich bereits in den Jahren 1985/86 zwölf Mutterhäuser in der Bundesrepublik und der damaligen DDR besucht und dort ca. 50 sog. "Feierabendschwestern“ in Gruppen- und Einzelgesprächen nach ihren Erinnerungen an das Dritte Reich befragt. Einige dieser Diakonissen im Ruhestand wurden durch meinen Besuch zum ersten Mal zu Aussagen über dieses Thema angeregt. Andere hatten bereits in ihrer Gemeinschaft über diese Zeit nachgedacht, keine von ihnen hatte jedoch vor meinem Besuch mit Personen, die außerhalb des Mutterhauses standen, über die NS-Zeit gesprochen. Alle Schwestern hatten die Zeit von 1933 bis 1938 als junge Frauen im aktiven Dienst als Gemeinde-, Kranken- oder Kindergartenschwester, als Lehrerin oder Erzieherin in Behindertenanstalten erlebt. Da die Interviews nur rudimentär in meine Dissertation eingegangen sind, will ich sie im folgenden ausführlicher zu Wort kommen lassen ${ }^{8}$. Zunächst möchte ich vier Geschichten wiedergeben, die sich mit den Jahren 1933 bis zum Kriegsbeginn beschäftigen.

1. Bei vielen Schwestern überwog in der Zeit von 1933 bis 1938/39 die Begeisterung für die sogenannte „nationale Erhebung“. So erzählte etwa Schwester Elise aus dem Mutterhaus Frankenstein in Schlesien: „Ich war damals noch jung, ich

\section{Lauterer, Liebestätigkeit 141-148.}

${ }^{8}$ Zur Problematik von mündlichen Interviews als Quellen der Geschichtsforschung vgl. Daniel Bertaux, Isabelle Bertaux-Wiame, Autobiographische Erinnerung und kollektives Gedächtnis, in: Lebenserfahrung und kollektives Gedächtnis. Die Praxis der „Oral History“, hrsg. von Lutz Niethammer (Frankfurt 1980) 108-122; Jürgen Straub, Zur narrativen Konstruktion von Vergangenheit. Erzähltheoretische Überlegungen und eine exemplarische Analyse eines Gruppengespräches über die NS-Zeit, in: Bios 9 (1996) 30-58; Robin Collmenn, Geron Henft, Autobiographisches Gedächtnis und aktuelle Lebensperspektive im Alter. Eine empirische Studie biographisch rekonstruierter Kriegserfahrungen, in: Bios 9 (1996) 59-73. 
hab mich in den ersten Jahren begeistert. Ich hab auch mal den Führer gesehen, in Neiße. Ich muß sagen, der Mann hatte eine dämonische Anziehungskraft. Das können Sie sich nicht denken. Wir standen ganz vorne, und er fuhr an uns vorbei. Jede von uns hatte den Eindruck: Er hat mich angeguckt. Ja, der hatte einen Blick - dämonisch - anders kann ich das gar nicht sagen. Ich meine, ich habe als Kind einmal Kaisers Söhne gesehen, in Liegnitz. Die waren da in der Grenadierkaserne, und da mußten wir von der Schule aus hin. Das war etwas ganz anderes. Die sah man, als wenn man einen großen Bruder sieht, so als hätte man ihnen um den $\mathrm{Hals}$ fallen wollen - das war etwas vollkommen anderes." "9

2. Eine Diakonisse aus Kassel berichtete über eine Begegnung mit Hitler ähnlich: „Ich hab ja auch Adolf Hitler erlebt. Das kann ich sagen, ich hab ihn ja gesehen. Ja, persönlich. Ja, er war einmal hier in Kassel, er fuhr durch die Königstraße und stand im Wagen. Und da haben wir alle Spalier gestanden. Das muß aber schon 1935/36 gewesen sein. Wissen Sie, als er kam, lag ja Deutschland sehr danieder. Die ganzen Arbeitslosen wurden ja plötzlich alle beschäftigt - und wir haben uns alle mitgefreut. Ich war damals schon im Mutterhaus. Ich bin 1933 eingetreten - mit dem Adolf bin ich angekommen - draußen hab' ich nichts erlebt. Und es wurden ja auch die ganzen Autobahnen gebaut als Arbeitsbeschaffung. Erst ging ja alles gut voran, und wir haben uns gefreut. Und dann, 1937, das weiß ich noch wie heute, war ja dann in Bückeburg das große Erntedankfest, wo er dann kam mit der ganzen Mannschaft, und da durften auch wir Seminaristinnen hin. Viele Schwestern in meiner Gruppe waren neidisch, daß ich da mitkonnte, das gehörte nämlich zu meiner Ausbildung, das war eine Studienfahrt. Um 6 Uhr früh haben wir gerade noch einen Platz an der Absperrlinie bekommen. Da standen schon die ganzen Ehrenformationen. Also das war ein Erleben, ich weiß das noch wie heut. Und die Schwestern haben gesagt: ,Du mußt dann für uns alle Heil rufen!'“10

3. Eine Diakonisse erinnerte sich an ihre Anfangszeit im Mutterhaus: „Ich bin dann als Schwesternschülerin aufgenommen worden, das war im Oktober 1932. Im Februar 1933, als der Reichstag in Brand stand, da war mein erster Ausflug. Nur da bekam ich frei. Wir vier Ausgehschwestern waren völlig unerfahren. Man war begeistert von den vielen Anhängern, alles sollte anders werden. Die vielen Arbeitslosen, die Brüder, die Verwandten, die sich dieser Sache angenommen hatten. Am 1. Mai 1933 - das ist einem unvergeßlich - die Welle von Menschen, die den Marktplatz überflutete. Und dann ging das so weiter, bis man dann als Schwester aufgenommen wurde, und dann ging alles ganz gut. Dann ging es auch nicht mehr lange, da kam ich nach Treysa ins Haus Hephata. Da wurde man als junge Schwester noch nicht so viel gewahr." ${ }^{11}$

9 Gespräch Heide-Marie Lauterer mit Schwester Elise Kunick, Mutterhaus Frankenstein, Schlesien, heute Wertheim (13.2. 1985).

10 Gespräch Heide-Marie Lauterer mit Schwester Margarete Baum, Mutterhaus Kassel (15. 2. 1986) $2 \mathrm{f}$.

11 Gespäch Heide-Marie Lauterer mit Schwester Katharina Happel, Mutterhaus Kassel (2. 5. 1986) 10. 
4. Eine andere Diakonisse war 1933 Gemeindeschwester in einer ländlichen Gemeindestation. Sie erzählte: „Ja, die Machtergreifung habe ich erlebt in Münchhausen bei Marburg. Es war eine sehr kirchliche Gemeinde, sehr stolze Bauern, die Kinder gingen alle noch in Tracht. Zuerst sah das so aus, als wäre das 'ne ganz gute Sache. Die Münchhäuser waren auch ganz davon angetan, denn die Nationalsozialisten, die zeigten sich kirchlich. Sie feierten das Erntedankfest immer sehr groß in der Kirche. Und es bildete sich auch eine NS-Frauenschaft, der ich mich aber nicht anschloß, weil ich von Anfang an gegen einiges Bedenken hatte. Obwohl es mir auch imponiert hat, wenn ich so sah, wie nach der Arbeitslosenzeit die Männer so schön geschlossen und in straffer Zucht die Diakonissenstraße herauf marschierten. Das habe ich vom Mutterhaus aus gesehen, das hat mich richtig beeindruckt. ${ }^{\text {}} 12$

Obwohl diese Geschichten selbstverständlich keinen repräsentativen Überblick über die Einstellungen der Kaiserswerther Mutterhausdiakonie zum NSRegime in der Anfangsphase geben, sind diese Zeugnisse in vieler Hinsicht bemerkenswert. Sie stammen von Frauen unterbürgerlicher, kleinbäuerlicher, kleinbürgerlicher Schichten, in wenigen Fällen auch von Töchtern aus Beamtenfamilien des gehobenen Dienstes, sowie vereinzelt von Frauen aus Pastorenfamilien. Es sind Zeugnisse, die über die Erinnerungen an die Selbstwahrnehmung und die Einstellung junger protestantischer Frauen in den ersten Jahren des NS-Regimes Aufschluß geben; sie sind in die Forschungsliteratur der Diakoniegeschichte bisher noch kaum eingegangen.

Von wenigen Ausnahmen abgesehen - Diakonissen, die von Anfang an eine NS-kritische Haltung zeigten und meistens aus dem Bildungsbürgertum stammten - begeisterte sich die Mehrheit aus den kleinbürgerlichen und kleinbäuerlichen Schichten für das Hitlerregime. Die Frauen erinnerten sich plastisch an die Faszination, die von dem Mann Hitler ausging. Sie konstruierten einen Gegensatz zu ihrer Wahrnehmung der Söhne des deutschen Kaisers. Für diese hatten sie geschwärmt, doch seien sie ihnen wie Brüder vorgekommen.

Im Kaiserreich hatte das Familienmodell der Mutterhausdiakonie seine Entsprechung in einem familialen Politikverständnis der Diakonissen gefunden ${ }^{13} . \mathrm{Im}$ Dritten Reich wandelte sich dieses Verständnis entsprechend der NS-Propaganda zu einem personalisierten Politikverständnis, das auf einen charismatischen Führer zielte ${ }^{14}$. Dieser bewährte sich sogar noch in der Rückschau: Die Schwestern bewunderten in ihrer Erinnerung immer noch die unmittelbar sichtbaren innenpolitischen Erfolge von damals - die vermeintliche Beseitigung der Arbeitslosigkeit und die Wiederherstellung der öffentlichen Ordnung. Und sie riefen sich noch einmal die Faszination vor Augen, die von den Massen ausging, die die NSDAP mobilisiert hatte.

12 Gespräch Heide-Marie Lauterer mit Schwester Aenne Linke, Mutterhaus Kassel (2. 5. 1986) 2.

13 Vgl. Lauterer, Liebestätigkeit 25.

14 Vgl. Ian Kershaw, Hitlers Macht. Das Profil der NS-Herrschaft (München 1992) 31 ff. 
Trotz allem blieben bei den Diakonissen Vorbehalte, die manche junge Schwester davon abhielten, sich freiwillig einer NS-Parteiorganisation anzuschließen. Diese Vorbehalte hielten jedoch Druck gegenüber nicht stand. Kam er von seiten der Partei, so fügten sie sich, wie das Beispiel der oben zitierten Schwester Aenne aus dem Mutterhaus Kassel zeigt. Als Leiterin eines evangelischen Kindergartens, der nicht im Mutterhaus, sondern in den Räumen der politischen Gemeinde beherbergt war, wurde sie vom Ortsgruppenleiter und Bürgermeister ihres Dorfes dazu aufgefordert, der NS-Frauenschaft beizutreten. Dieser Aufforderung kam sie nach. Sie berichtete: „Ich übernahm in Heringen den Kindergarten. Da war der Bürgermeister Ortsgruppenleiter. Und das Haus, in dem der Kindergarten und auch die Schwesternstation war, gehörte der politischen Gemeinde. Nur die Arbeit, die darin geschah, das war kirchliche Arbeit. Und der Bürgermeister in der Person des Ortsgruppenleiters hat mich dann immer mal bestellt. Zunächst hat er mich gefragt, warum ich nicht in der Frauenschaft sei und daß das doch sehr wünschenswert wäre. Und das hat mich dazu veranlaßt, der Frauenschaft beizutreten." Schwester Aennes Einstellung zur NS-Ideologie blieb distanziert, aber sie behielt ihre Kritik für sich oder nahm sie im nachhinein zurück. „Ich erinnere mich daran, daß bei einer Feier eine Frau ein Gedicht aufgesagt hat. Das war eine Frau der Frauenschaft, und da schloß jeder Vers mit dem Satz: ,Ich habe dem Führer in die Augen geschaut.' Sehen Sie - na ja ... Und im Kindergarten habe ich es dann auch zu spüren bekommen. Ich hatte außerdem Kindergottesdienst zu halten, und im Kindergottesdienst wurde ich bespitzelt. Und wurde nach einem Kindergottesdienst dann auch zum Bürgermeister bestellt. Da hatte ich eine Bemerkung gemacht. Es war wirklich sehr dumm, ich muß es selber sagen. Ich hatte nämlich beim Gleichnis vom barmherzigen Samariter einen Vergleich gezogen. Ich hatte gesagt - alle gehen vorbei an diesem unter die Räuber gefallenen SS-Mann oder Ortsgruppenleiter - aber der, der ihm geholfen hat, das war ein Jude. Ja, ein Jude, habe ich gesagt. Das war sehr riskant - ich weiß das jetzt hinterher." 15

Solche Erfahrungen änderten nichts daran, daß aus den Erzählungen dieser und anderer Diakonissen über die Anfangszeit des NS-Regimes Zufriedenheit und Einverständnis mit der politischen Lage spricht; die Schwestern blickten voller Optimismus in die Zukunft. Sie fühlten sich als Teil des deutschen Volkes, mit dem es nun wieder aufwärts ging. Doch stellte sich die Situation in Deutschland etwa für den jüdischen Bevölkerungsteil bereits während der Machtübernahme völlig anders dar. Dies zeigt schlaglichtartig ein Tagebucheintrag Viktor Klemperers vom März 1933. Er schrieb: „Inzwischen im Innern kein Blutvergießen, aber Bedrückung, Bedrückung, Bedrückung. Niemand atmet mehr frei, kein freies Wort, weder gedruckt, noch gesprochen. Nichts mehr von mir erscheint." 16

15 Gespräch Heide-Marie Lauterer mit Schwester Aenne Linke, Mutterhaus Kassel (2. 5. 1986) 2.

16 Viktor Klemperer, Ich will Zeugnis ablegen bis zum letzten, Bd. 1 (Berlin 1995) (27. März 1933) 15. 
Wie ich in meiner Dissertation ${ }^{17}$ dargelegt habe, herrschte 1933 auch bei den beiden Vorsitzenden des Kaiserswerther Verbandes, Hans Lauerer und Siegfried Graf von Lüttichau, Einverständnis mit der politischen Wende. Die Männer zeigten sich vor allem empfänglich für das nationale, militärstrotzende und kirchliche Pathos, wie es erstmalig am "Tag von Potsdam" in Szene gesetzt wurde. Wie die beiden Vorsitzenden, so glaubte die Mehrheit der Mutterhausvorsteher, in dieser Inszenierung die Auferstehung des Kaiserreiches zu erkennen. Dabei ignorierten sie die ebenfalls zur Schau gestellte NS-Ideologie, wie sie im neuen Staatsemblem, dem Hakenkreuz, zum Ausdruck kam, das am 21. März 1933 in der Krolloper den Raum beherrschte. Göring hatte dessen Sinngehalt in seiner Eröffnungsrede im Reichstag, kurz vor der Annahme des Ermächtigungsgesetzes, interpretiert. Es sei das „uralte Zeichen unserer Vorväter, und doch ewig verjüngend, ewig neu, das Sonnenzeichen, als Zeichen des Aufstieges, als Zeichen der Reinheit, als Zeichen der Ehre" 18 . In der Kaiserswerther Mutterhausdiakonie fragte man kaum nach den politischen und pseudoreligiösen Inhalten dieses Symbols, sondern begnügte sich damit, daß Hitler in seiner Regierungserklärung Verbeugungen gegenüber der christlichen Religion gemacht hatte, die freilich vorwiegend als Zugeständnisse an die katholische Zentrumspartei gedacht waren.

Auf meine Frage, ob sie sich in der Zeit vor 1933 für Politik interessiert hätten, antworteten die meisten Diakonissen mit einem klaren „Nein“. Als Grund dafür nannten sie rückblickend chronischen Zeitmangel. Es mag sein, daß einige Schwestern damit in der Rückschau ihr generelles Desinteresse an Politik rechtfertigen wollten. Doch muß man hinzufügen, daß nur sehr wenige Mutterhausvorstände in der Weimarer Republik darauf geachtet hatten, daß den Diakonissen im Berufsalltag Zeit für politische Bildung eingeräumt wurde. So verstanden die Oberinnen des Verbandes, die oft aus bürgerlichen Familien oder aus dem Adel stammten, mehrheitlich ihr Amt als unpolitisch. So plädierte Oberin Anna von Ehrenstein 1931 für den Austritt des Kaiserswerther Verbandes aus der Vereinigung der Evangelischen Frauenverbände mit folgender Begründung: „Die Probleme auf politischem, rechtlichem, medizinischem und sozialem Gebiet, die dort verhandelt werden, liegen abseits vom Dienst der Mutterhäuser und der Schwestern."19

Andererseits zeigen die Geschichten der einstmals jungen Probeschwestern jedoch, wie eng diese Frauen in der Rückschau auf 1933 ihre individuelle Biographie mit politischen Ereignissen koppelten. So geht der Bericht über die Feier des Ersten Mai 1933 in der dritten Erzählung direkt zur Ausbildung der jungen Schwester über. Diese verlief ebenso gut wie das politische Leben außerhalb des Mutterhauses. Ich zitiere noch einmal: „Man war begeistert von den vielen Anhängern, alles sollte anders werden. Die vielen Arbeitslosen. Die Brüder, die Verwandten, die sich dieser Sache angenommen hatten. Am 1. Mai 1933, das ist einem unver-

17 Lauterer, Liebestätigkeit 38-48, 70-73.

18 Verhandlungen des Reichstags, Stenographische Berichte, Nr. 457, 1. Sitzung (21.3.1933) 17.

19 Vgl. Lauterer, Liebestätigkeit 40, Anm. 98. 
gesslich - die Welle von Menschen, die den Marktplatz überflutete. Und dann ging das so weiter, bis man dann als Schwester aufgenommen wurde, und dann ging alles ganz gut." 20

Die positive Beurteilung der innenpolitischen Lage beschränkte sich dabei nicht nur auf die Zeit unmittelbar nach der Machtergreifung, sondern erstreckte sich auf die folgenden fünf Jahre. Glaubt man den Erzählungen, so schien der Arbeitsalltag in der Zeit von 1933 bis 1938 im Mutterhaus und auf den Gemeindestationen ohne Störungen verlaufen zu sein. Für diese Annahme spricht, daß die Erinnerungen der Schwestern an die ersten fünf Jahre des NS-Regimes, abgesehen von den spektakulären Aufmärschen, wenige konkrete Ankerpunkte fanden.

So vermitteln die Interviews mit den Schwestern an der Verbandsbasis denselben Eindruck, der sich bereits aus der Untersuchung des Verhaltens des Verbandsvorstandes ergeben hat - daß sich die Mutterhausdiakonie in der Zeit von 1933 bis 1938 unter der Maßgabe der Bestandssicherung und der Bewahrung ihrer traditionellen Arbeitsstrukturen im Bereich der Wohlfahrtspflege ohne größere Konflikte in das NS-Regime einfügte. Das Außerkraftsetzen der Grundrechte, die Verfolgung und Mißhandlung politischer Gegner, die ersten antijüdischen Gesetze, das Gesetz zur Verhütung erbkranken Nachwuchses mit seinen unmittelbaren Konsequenzen für die konfessionellen Krankenhäuser, die nun auch Zwangssterilisierungen durchführen mußten, die Errichtung der Konzentrationslager, all das kommt in den Interviews nicht oder nur vereinzelt und am Rande vor. Es wurde entweder nicht wahrgenommen, oder verdrängt, oder aber als notwendige Kehrseite der wirtschaftlichen und sozialen Stabilisierung hingenommen.

Dies zeigt schlaglichtartig die Erinnerung einer Schwester aus Königsberg an einen arbeitslosen Mann in ihrer Gemeinde. Sie berichtete: „Sie nannten sie damals Faulenzer. Die wurden dann abgeholt und kamen dann in eines dieser Lager. Wir wußten nicht, wohin sie kamen - und das waren ja Arbeitslager; dabei - es gab ja gar nicht genug Arbeit für alle. Als der Mann zurückkam, erzählte der mir dann einiges. Die hatten ja Schweigepflicht, aber mir hat er doch einiges erzählt. Wenn ein Mann ein Paket bekam, mußte er es sich bei der Verwaltung abholen und es auf allen Vieren kriechend mit den Zähnen tragen. Sie wurden geschlagen und hatten schlechte Verpflegung. " 21

Ich will im folgenden an drei Beispielen zeigen, welche Aspekte ihres Arbeitsalltags die Schwestern in ihren Geschichten rückblickend hervorhoben.

1. Eine Diakonisse aus dem Mutterhaus Kassel war Leiterin eines mutterhauseigenen Kindergartens. Sie berichtete: „Ich habe meinen Kindergarten nach 1933 geführt wie bisher. Da hat uns niemand reingesprochen. Im Kindergarten, da hatten wir nichts zu erfüllen auf nationalsozialistischer Ebene. Wir haben den Kindergarten geführt wie immer. Wir haben unseren Morgenkreis gemacht, haben ein

20 Gespräch Heide-Marie Lauterer mit Schwester Katharina Happel, Mutterhaus Kassel (2. 5. 1986) 10.

21 Gespräch Heide-Marie Lauterer mit Schwester Berta Zimmer, Diakonissenmutterhaus Königsberg, heute Altenberg bei Wetzlar (28. 9. 1986). 
Lied gesungen. Haben mit den Kindern gebetet vor dem Essen und nach dem Essen und haben jede Woche eine biblische Geschichte erzählt. Und sonst haben wir natürlich auch die Kinder zu echten deutschen Kindern erzogen. Ja, wir haben marschiert und dann haben wir gesungen: ,Links, rechts, links rechts, Säbel an der Seite, schultert das Gewehr, mit welcher Freude ziehen wir daher.' So kleine Kinderlieder. Heute würde man sagen, das war militaristisch. Aber - das war ja noch so richtig Erziehung, so wie die Schule. Und diese Lieder - damit hatte man die Kinder ja auch in Zucht, die machten das gerne. Aber nicht, daß uns jetzt jemand gesagt hätte, so müßt Ihr das machen. Es war unser Kindergarten. "22

2. Im Kindergärtnerinnenseminar dieses Mutterhauses, das neben Diakonissen auch junge protestantische Frauen von außerhalb ausbildete, wurde Hitlers „Mein Kampf" gelesen und Rassenkunde unterrichtet. Darüber hinaus wurde als Prüfungsvoraussetzung von allen Schülerinnen, die Diakonissen eingeschlossen, der Arierausweis verlangt.

Daß diese Regelung offenbar ausgeführt wurde und die Diakonissen noch im nachhinein einen möglichen inneren Widerstand zu überwinden suchten, indem sie versuchten, dieser Regelung etwas Positives abzugewinnen, zeigt die Aussage einer Kasseler Diakonisse: „Nun ja, man mußte sich ausweisen, daß man ein deutscher Mensch war, arische Abstammung bis zum Urgroßvater. Ja, und das war auch wiederum interessant, so eine kleine Ahnenforschung. " 23 Zum Thema Ariernachweis berichtete eine andere Diakonisse, deren Vater Beamter war und deshalb seine Abstammung offenlegen mußte, daß ihr Vater gleich mehrere Durchschläge des Nachweises für alle Kinder habe anfertigen lassen. Die Diakonisse kommentierte: „Ja, das war ja gut für die Kinder. Ja, das mußte man ja haben. Sonst konnte man ja kein Staatsexamen machen. Ja, und überhaupt, das mußte gemacht werden." 24

3. Das Frankfurter Diakonissenmutterhaus beteiligte sich im Mai 1934 an der Rhein-Mainischen „Braunen Messe“. Die Diakonissen arbeiteten an der Sonderausstellung der NS-Frauenschaft: „Die deutsche Frau beim Aufbau des Dritten Reiches" mit. Sie gaben einen Überblick über Organisation, Tagesablauf und Arbeitsgebiete der Diakonissen sowie über die Ausbildung in der Mutterhausdiakonie. Als anschauliches Beispiel bauten sie einen ländlichen Kindergarten auf. Dabei achteten die Diakonissen darauf, das „Einzigartige“ ihrer Arbeit zu zeigen, das im Mutterhaus Frankfurt etwa in einer Paramentikwerkstatt bestand. Die Diakonissen waren der Ansicht, die Ausstellung der NS-Frauenschaft „sei etwas für das Volk gewesen, die NSV habe da etwas für die Volksbildung “ getan. In diesem Bereich arbeitete das Mutterhaus auch außerhalb der Ausstellung mit der

22 Gespräch Heide-Marie Lauterer mit Schwester Margarete Baum, Mutterhaus Kassel (15. 2. 1986) $5 \mathrm{f}$.

23 Gespräch Heide-Marie Lauterer mit Schwester Margarete Baum, Mutterhaus Kassel (15. 2. 1986) 5 f.; Gespräch Heide-Marie Lauterer mit Schwester Katharina Happel, Mutterhaus Kassel (5.2. 1986) 22.

24 Gespräch Heide-Marie Lauterer mit Schwester Katharina Happel, Mutterhaus Kassel (5. 2. 1986) 22. 
NSV zusammen. So stellte das Frankfurter Diakonissenhaus beispielsweise seine Lehrküche für Kochgruppen der NSV zur Verfügung25.

Aus diesen Erzählungen gehen drei Bedingungen hervor, die den Schwestern die Kooperation mit den Institutionen und Behörden des NS-Regimes in der hier interessierenden Zeit ermöglichten.

1. Die traditionelle, religiöse Form sowie die Inhalte des Diakonissendienstes blieben gewahrt. Im Kindergarten beispielsweise wurde gebetet, gesungen und biblische Geschichten erzählt; die Räume wurden durch religiöse Symbole geschmückt - selbst auf der Braunen Messe stellten die Diakonissen u.a. sakrale Textilien aus, die sie in ihrer Paramentikwerkstatt herstellten - und der Tagesablauf sowie das Jahr wurde auch nach 1933 von religiösen Ritualen strukturiert.

2. Einzelne Elemente nationalsozialistischer Pädagogik, wie die frühkindliche militaristische Erziehung, waren durchaus mit der Praxis in den evangelischen Kindergärten vereinbar. Darüber hinaus entsprach die Betonung der Volksgemeinschaft, ausgedrückt in der Devise: Gemeinnutz geht vor Eigennutz, dem vormodern-religiösen Gemeinschaftsdenken der Diakonissen.

3. Die Diakonissen leisteten behördlichen Anordnungen, wie der Auflage des Ariernachweises als Voraussetzung für die Abschlußprüfung der evangelischen Kindergärtnerinnen, unwidersprochen und mit einem gewissen Verständnis Folge. Die Mutterhausleitungen, die Konflikte mit der Obrigkeit vermeiden wollten, sorgten ihrerseits für die Einhaltung dieser Anordnungen. Im Mutterhaus Kaiserswerth führte dies dazu, daß zwei Diakonissen, die auf einem amtlichen Meldebogen gewissenhaft über ihre jüdische Herkunft Auskunft gegeben hatten, nach Theresienstadt und Auschwitz verschleppt wurden. Nur eine von ihnen kehrte zurück ${ }^{26}$.

Eine solche zumeist unreflektierte Bereitschaft, im Bereich der Wohlfahrtspflege mitzuarbeiten, die gleichwohl Raum für mentale Vorbehalte bot, fand sich vor allem bei den Schwestern an der Verbandsbasis sowie bei der Mehrheit der Mutterhausleitungen. Auf der Ebene der übergeordneten Organisation evangelischer Schwesternverbände, der "Diakoniegemeinschaft“ dagegen war die Begeisterung im Untersuchungszeitraum bewußt und ungeteilt. Ich habe in meiner Dissertation ausführlich über die institutionelle Einbindung dieser 1933 gegründeten Dachorganisation in das System nationalsozialistischer Wohlfahrts- und Gesundheitsorganisationen sowie über die politische Mentalität ihrer Führerin, Auguste Mohrmann, geschrieben 27. Mohrmann war in Personalunion „Verbandsoberin" des Kaiserswerther Verbandes nach dessen Umstrukturierung im Frühjahr 1933. Als einziges weibliches Mitglied des dreiköpfigen „Führerrates" war sie im Mai 1933 in die NSDAP eingetreten ${ }^{28}$. An dieser Stelle möchte ich zeigen,

25 Vgl. Lauterer, Liebestätigkeit 191.

26 Vgl. Ruth Felgentreff, "Ist verpflichtet, den Judenstern zu tragen“. Eine Dokumentation über Johanne und Erna Aufricht. Kaiserswerth, Theresienstadt, Auschwitz (Kaiserswerth 1973).

27 Vgl. Lauterer, Liebestätigkeit 77-82.

28 Mitgliedsnummer 3077449. Berlin, Document Center. Akte Auguste Mohrmann. 
daß spezifische Elemente des Selbstverständnisses dieses evangelischen Schwesternverbandes mit der nationalsozialistischen Volkstumsideologie übereinstimmten.

In einem Rundbrief der Diakoniegemeinschaft im Jahr 1935 bestimmte die „Führerin“, Auguste Mohrmann, die Rolle und Aufgabe der konfessionellen Schwestern im Dritten Reich. In direkter Anrede legte sie die Schwestern darauf fest, "Glied einer großen Kette zu sein, Teil einer Gemeinschaft, die aus dem gleichen Boden ihre Kräfte nimmt. Du dienst mit vielen Tausenden dem gleichen Herrn, Jesus Christus. “29 Sie fuhr fort: „Schwestern der Diakonie sind immer und überall Dienerinnen in und an ihrem Volk. Schwestern der Diakonie stehen neben den Schwestern der anderen Verbände im gleichen Kampf um die Gesund- und Reinerhaltung der Glieder des deutschen Volkes. Sie kämpfen inmitten einer großen Schar deutscher Frauen um neue Wertung von Tugenden, die im deutschen Volk weithin an Geltung verloren hatten: Opfer, Ehre, Treue, Gehorsam. Sie können und wollen nicht gleichgültig zuschauen in dieser Zeit der Entscheidungen, wo deutsches Volk in Kampf und Not neu geformt wird. Schwestern der Diakonie versuchen an ihrem bescheidenen Teil in Gehorsam, Hingabe und Treue das gigantische Aufbauwerk des Führers im Deutschen Volke zu unterstützen. “30

An diesem Text sind drei Aspekte bemerkenswert. Obwohl Diakonissen angesprochen werden, entsprechen die Zuschreibungen nicht den weiblichen Eigenschaften der Geschlechterrollenstereotypen, wie sie im Familienmodell der Mutterhausdiakonie seit dem 19. Jahrhundert für Männer und Frauen festgelegt waren und praktiziert wurden. Danach verstanden sich und wurden die Diakonissen verstanden als mütterlich, friedliebend, schützend und warmherzig, dabei den Männern, Vätern und Mutterhausvorstehern untergeordnet. Aus dieser Zuschreibung abgeleitet war der Arbeitsbereich der Diakonissen. Sie waren für den inneren, häuslichen, pflegenden, erzieherischen Bereich zuständig31.

Im Rundschreiben der Diakoniegemeinschaft zählen Frauen wie Männer als Volksglieder, als Teil des Ganzen, der Volksgemeinschaft. Explizit wird darauf hingewiesen, daß es für die deutschen Frauen und Diakonissen jetzt um eine „neue Wertung von Tugenden“ geht. Nämlich darum, „Opfer, Ehre, Treue und Gehorsam" neu zu verstehen. Verlangt wurde damit nichts anderes, als daß sich die Frauen diese Werte, die zum Arsenal des männlichen, militärischen Ehrenkodexes gehörten, zu eigen machen sollten.

${ }^{29}$ Auguste Mohrmann, Rundschreiben der Diakoniegemeinschaft 1935, Archiv des Kaiserswerther Verbandes. Akte Diakoniegemeinschaft; im folgenden zitiert: Rundschreiben.

30 Ebd.

31 Die Oberinnen waren für die Kommunikation unter den Schwestern zuständig. Im Verantwortungsbereich der Vorsteher lag dagegen die Verbindung zur Außenwelt sowie die Verbindung zum Überirdischen. Sie hielten Gottesdienst und informierten die Diakonissen über die Welt; sie schlossen Arbeits- und Kaufverträge ab, sie regelten die Finanzen des Mutterhauses. Den Zuschreibungen der männlichen Geschlechterrolle zufolge waren sie rational veranlagt, zuweilen auch hart und kämpferisch und den Frauen übergeordnet. 
Dies zeigt sich in der Rhetorik, die seit 1933 die dienenden und pflegenden Tätigkeiten von Frauen in Termini von Kampf faßt. Vor der "Volksgemeinschaft“", dem „Gigantischen Aufbauwerk des Führers“, glichen sich die Geschlechterrollen aneinander an und gingen in militärisch-soldatischen Tugenden auf, die für beide Geschlechter Gültigkeit erlangten ${ }^{32}$.

Diese Veränderung der Sprache korrespondierte mit der zunehmenden Militarisierung der Gesellschaft, die innerhalb des Kaiserswerther Verbandes schon im März 1933 zur Kenntnis genommen worden war. Eine Anfrage an den Verbandsvorstand hatte gezeigt, daß einzelne Mutterhausvorstände zu dieser Zeit mit der Aufstellung eines neuen Heeres rechneten und die Verwendung von Diakonissen als Krankenschwestern darin sicherstellen wollten ${ }^{33}$.

Die Radikalität, mit der nicht nur in dem zitierten, sondern auch in anderen Texten Diakonissen als Kämpferinnen für die Volksgemeinschaft angesprochen werden, ist nicht nur deshalb bemerkenswert, weil sie dem Mutterbild scheinbar diametral gegenüber stand. Sie sucht sogar in der nationalsozialistischen Ideologie der Zeit nach 1933 ihresgleichen, da das offizielle Frauenbild, wie es Gertrud Scholtz-Klink ${ }^{34}$ seit ihrer Einsetzung als Führerin der Reichsfrauenschaft im $\mathrm{Fe}$ bruar 1934 verkörperte und propagierte, stärker auf die Aufgaben und Eigenschaften deutscher Mütter sowie auf den Aufbau der Mütterarbeit abhob ${ }^{35}$.

Beim näheren Hinsehen erweisen sich die beiden Stränge jedoch als miteinander vereinbar. So etwa schrieb Lydia Gottschewskij ${ }^{36}$, die von Februar bis Juni 1933 den BDM sowie bis September die Reichsfrauenschaft geleitet hatte, im Jahr 1935: „Neben das Bild des Kämpfers tritt, noch schattenhaft in den Umrissen, aber erfüllt von treibenden Kräften, von Möglichkeiten künftiger Gestaltung, das Bild der Mutter. Und erst, wenn beide Bilder groß und leuchtend über unserem Volke stehen, jedes sicher in seinem Gefüge, stark und ruhig in sich selbst, aber eines das andere beschenkend mit seinen besten Kräften, ist unser Volk in seiner gültigen, wesenhaften Ordnung. “37 Aus diesem Zitat geht hervor, daß die Frauen an der "Volkwerdung" komplementär beteiligt sein sollten.

Wie sich NS-Führerinnen diese Teilhabe von Frauen vorstellten, wird bei Guida Diehl deutlich ${ }^{38}$. Sie galt 1932 als Aspirantin auf das Amt der Reichsfrauen-

32 Rundschreiben.

33 Vgl. Lauterer, Liebestätigkeit 80, 116.

34 Vgl. NS-Frauenbuch. Herausgegeben im Auftrag der obersten Leitung der NSDAP-Frauenschaft (München 1934) $15 \mathrm{ff}$.

35 Gertrud Scholtz-Klink, Deutsches Muttertum. Muttertag 1942, in: NS-Frauenwarte 18 (1941/42) 272, zitiert nach Leonie Wagner, Nationalsozialistische Frauenansichten, Vorstellungen von Weiblichkeit und Politik führender Frauen im Nationalsozialismus (Frankfurt 1996) 85. Im folgenden zitiert: Wagner, Nationalsozialistische Frauenansichten. ScholtzKlink schrieb 1942: „Mit jedem Kinde, dem eine Mutter das Leben schenkt, trägt sie zur Zukunft der Nation bei und wächst damit über sich selbst hinaus."

36 Zu ihrer Biographie vgl. Wagner, Nationalsozialistische Frauenansichten 190-196.

${ }^{37}$ Lydia Gottschewski, Von der Formkraft der Bilder, in: Deutsche Frauenkultur 6 (1935), zit. nach Wagner, Nationalsozialistische Frauenansichten 84.

${ }^{38} \mathrm{Zu}$ ihrer Biographie vgl. Wagner, Nationalsozialistische Frauenansichten $188 \mathrm{ff}$. 
führung und versuchte nach 1933, ihre Vorstellungen in einer Frauenabteilung der „Deutschen Christen“ unterzubringen. Für Diehl war jedes Todesopfer eines deutschen Mannes mit dem „Lebensopfer" einer deutschen Frau verbunden. An solche Vorstellungen knüpfte Auguste Mohrmann 1933 an. Die Führerin der Diakoniegemeinschaft ging aber noch einen Schritt weiter, indem sie auch die Mütter als eines Todesopfers für würdig erachtete. Der bisher sinnlos erscheinende Tod der Söhne im Ersten Weltkrieg wurde angesichts des Dritten Reiches rückblikkend zum „Heldentod“ und das erneute Gebären und Erziehen von Knaben konnte verstanden werden als „heiliger Dienst“, $d$.h. als Dienst an der Volksgemeinschaft und als Kriegsdienst. „Deutsche Mütter", so rief Auguste Mohrmann aus, „die Ihr dem Vaterlande Söhne schenkt und erzieht, Gott segne Euch und Euren heiligen Dienst." 39

Als die NSDAP im März 1934 damit begann, eine eigene Schwesternorganisation aufzubauen, war es diese ideologische Nähe auf der Ebene der Sekundärtugenden, d.h. des Dienstes, die zwischen den Ideen führender Frauen der Mutterhausdiakonie und den Führerinnen der NS-Frauenschaft bestand, die es der NSDAP erlaubten, in entscheidenden Punkten auf das Kaiserswerther Mutterhausmodell als Vorbild zurückzugreifen.

So übernahm die NSV für die neue politisch-soziale Berufsorganisation für Frauen den aus dem kirchlich-religiösen Bereich stammenden und pietistisch geprägten Begriff „Schwesternschaft“. Die Frauen entschlossen sich, in diese Schwesternschaft „einzutreten“ und wurden in sie „aufgenommen “40. Die NS-Schwestern erhielten wie die Kaiserswerther Diakonissen eine Tracht, die sich nur in der Farbe braun vom dunkelblauen Kaiserswerther Markenzeichen unterschied. Sonn- und feiertags sollte ein dunkelbraunes Wollkleid und ein gleichfarbener Mantel getragen werden. Wie in Kaiserswerth gehörte eine weiße Haube dazu, die sonntags mit einem braunen Haubentuch getragen wurde ${ }^{41}$. Die Tracht stellte nicht nur eine einfache und zweckmäßige Arbeitskleidung dar, sondern sie war ein „Ehrenkleid“. Selbst die Bezahlung richtete sich nach dem Vorbild der Mutterhäuser. Die NS-Schwestern erhielten freien Lebensunterhalt, freie Tracht sowie ein nach Dienstjahren gestaffeltes monatliches Taschengeld. Wie ihre kirchlichen Schwestern waren sie der Steuerpflicht enthoben. Mit dem Eintritt in die NSSchwesternschaft war der Beitritt zu einer Altersversicherung verbunden, die die Schwesternschaft übernahm ${ }^{42}$. Die Analogien gingen noch weiter, denn für die NS-Schwesternschaft wurde ein sogenanntes „Mutterhaus“ eingerichtet. Es war dem Rudolf-Hess-Krankenhaus in Dresden angegliedert und diente der weltanschaulichen und beruflichen Schulung ${ }^{43}$.

39 Christliche Kinderpflege 41 (1933) 193.

40 NSDAP. Schwesternschaft der NSV. Anordnung Nr. 1. 28. 3. 1934. BA Koblenz. NS 37/

1040, im folgenden zitiert: Anordnung Nr. 1

41 Anordnung Nr. $1,4$.

42 Anordnung Nr. 1, 2.

${ }^{43}$ NSDAP. NS-Schwesternschaft. Anordnung Nr. V 2/34. (8. 6. 1934) 1. 
Wie in Kaiserswerth unterlagen die NS-Schwestern dem "Sendungsprinzip“. Dies geht aus der im Jahre 1935 eingeführten Eidesformel hervor. Sie enthielt die Verpflichtung, ,an jedem Platz, an den ich gestellt werde, meine Berufsaufgaben als nationalsozialistische Schwester treu und gewissenhaft im Dienst der Volksgemeinschaft zu erfüllen“. Die Formel, die mit dem Treueschwur auf Adolf Hitler begann, endete mit den Worten: "So wahr mir Gott helfe" 44 . Die Schwesternschaft der NSV erhielt den offiziellen Auftrag, „zur Schaffung der wahren Volksgemeinschaft" beizutragen - eine Aufgabe, der sich auch die Diakonissen verpflichtet fühlten ${ }^{45}$.

Während die Diakonissen jedoch auch im Dritten Reich ihren Dienst christlich begründeten, verlangte die NSV von ihren Schwestern, daß sie ihre Aufgaben auf dem Gebiet der Krankenpflege im Sinne der NSDAP ausführten ${ }^{46}$. Damit war ihr Dienst ausdrücklich politisch legitimiert und für politische Belange instrumentalisiert. So wurde als Eintrittsvoraussetzung bei der NSSchwesternschaft politisch-ideologisch korrektes Verhalten verlangt. Es konnten nur solche Frauen aufgenommen werden, die sich bereits vor dem 30. Januar 1933 nationalsozialistisch betätigt hatten, oder solche Frauen, deren Familien bereits vor dieser Zeit nationalsozialistisch aktiv waren ${ }^{47}$. In die Kaiserswerther Schwesternschaften durften nur Frauen protestantischen Glaubens eintreten, und die Mutterhausvorsteher verlangten ein pfarramtliches Führungszeugnis von den Novizen. Der entscheidende Unterschied zwischen dem Dienst an der Volksgemeinschaft, wie ihn die Kaiserswerther Diakonissen verstanden, und dem Dienst an der Volksgemeinschaft im Sinne der NSV lag in der nationalsozialistischen Rassenideologie begründet. Nur rassisch wertvolle, erbgesunde und arische Menschen sollten überhaupt in den Genuß der Leistungen der NSVolkswohlfahrt gelangen.

Solche Kriterien standen freilich dem Dienstverständnis der Kaiserswerther Diakonie diametral entgegen: Die Kaiserswerther Diakonissen verstanden ihren Dienst als Nachfolge Christi, der allen notleidenden Menschen zugute kommen sollte. "Wir haben alle gepflegt", so antwortete mir eine Krankenschwester, "wer immer da krank war, ob das Jude oder Heide oder Katholik war oder Protestant, das war ganz egal. " ${ }^{48}$ Aber sie vergaß hinzuzufügen, wie lange es ihr gelungen war, diesem Auftrag treu zu bleiben.

Wenn die NSDAP gerade die traditionellen Formen der Mutterhausdiakonie partiell übernahm, um deren Erhaltung die „Führer des Kaiserswerther Ver-

44 NSDAP. NS-Schwesternschaft. Anordnung Nr. 7 (11.11. 1935) BA Koblenz NS 37/1040.

45 Dienstanweisung für die NS-Gemeindeschwestern, in: Herwart Vorländer, NSV. Darstellung und Dokumentation einer nationalsozialistischen Organisation (Boppard 1988) 310, Dokument Nr. 119, im folgenden zitiert: NSV.

46 Ebd. 310.

47 Ebd. 2.

48 Gespräch Heide-Marie Lauterer mit Schwester Katharina Happel, Mutterhaus Kassel (2. 5. 1986). 
bandes" so sehr rangen ${ }^{49}$, so zeigt sich daran einerseits, wie eng diese aus dem 19. Jahrhundert stammenden Formen sogar in der NS-Öffentlichkeit mit der Vorstellung qualitätsvoller Krankenpflege und sozialer Fürsorge verbunden waren. Andererseits konnte die NSV gerade in der Konsolidierungsphase des Dritten Reiches die weitverbreitete Arbeit der Mutterhausdiakonie auf dem Gebiet der Krankenpflege nicht ersetzen oder gar auf sie verzichten.

$\mathrm{Daß}$ die NSV dennoch nach eigenen Prägungen des Schwesterndienstes suchte, zeigt ein Werbeslogan, der moderne junge Frauen für den Schwesternberuf interessieren sollte. Es war vor allem die Ehe- bzw. Kinderlosigkeit, die nicht in das nationalsozialistische Frauenbild paßte. In dem Werbespot, an den sich der Präsident des Centralausschusses für Innere Mission, Pastor Konstantin Frick, nach 1945 erinnerte, hieß es: „Schwester Erika pflegt, schwimmt, reitet, spielt Tennis und schenkt dem Führer ein Kind. “50 An diesem Slogan fällt auf, daß gerade der politisch-weltanschauliche Auftrag, der in den Dienstanweisungen der NS-Schwesternschaft an erster Stelle stand, fehlte. Darüber hinaus sprach dieses Bild eher die Lebensweise einer privilegierten (Ehe-)Frau in einer utopischen Freizeitgesellschaft an, ja es kam sogar in die Nähe der von den Nationalsozialisten so sehr geschmähten „Luxusweibchen“; dem Selbstverständnis einer modernen berufstätigen Frau oder gar einer verantwortungsbewußten Krankenschwester entsprach der Slogan jedenfalls nicht. In anderen Werbetexten wurde auf den Unterschied zwischen den konfessionellen Schwestern und den jugendlichen NS-Schwestern ausdrücklich hingewiesen. So hieß es im Berliner Tageblatt vom 26. März 1933: „Es ist wohl kein größerer Gegensatz zu denken als zwischen der krankenpflegerischen Schwester, der Nonne oder Diakonisse im faltenreichen Habit oder dem sportgestählten Mädel von 1937, das den Beruf der NS-Schwester wählt. " 51

Betrachtet man die Entwicklung der NS-Schwesternschaft in den Jahren 19341940 , so war ihr schon rein numerisch keine große Wirkung beschieden.

Einer offiziellen Statistik der NSV zufolge zählte die NS-Schwesternschaft im Jahre ihrer Gründung 1001 Schwestern. Im Jahre 1936 hatte sich die Zahl mehr als verdreifacht und war auf 3866 Schwestern angestiegen. Im Jahr 1938 war diese Zahl bereits wieder weit unter 3000 auf 2531 Schwestern gesunken. Selbst in den folgenden Jahren, als die NSV verstärkt begann, konfessionelle Schwestern aus ihren Gemeindestationen und Kindergärten zu verdrängen und mit eigenen Kräften zu besetzen, stieg die Zahl der NS-Schwestern nur langsam an und erreichte erst 1940 die Zahl 3524. Verglichen mit den evangelischen Schwesternschaften, die in der von Auguste Mohrmann geführten Diakoniegemeinschaft organisiert waren und 1933 über 50000 Schwestern zählten, war dies ein klägliches Häuflein.

49 Vgl. Lauterer, Liebestätigkeit 49-59.

50 Zit. nach Liselotte Katscher, Krankenpflege und Drittes Reich. Der Weg der Schwesternschaft des Evangelischen Diakonievereins 1933-1939 (Stuttgart 1990) 138, im folgenden zitiert als Katscher, Krankenpflege.

51 Katscher, Krankenpflege 138. 
Gerade die von der NSV umworbenen jungen "sportgestählten“ Mädel ließen sich schwerlich von dem Berufsbild der NS-Schwester, das zwischen zwei Polen, dem (konfessionellen) Dienstverständnis, verkörpert im Mutterbild, und der Vorstellung einer modernen Berufstätigkeit, hin- und herschwankte, anziehen. Wenn sich die Diakonissen der Mutterhausdiakonie vor allem aus den protestantischen, unterbürgerlichen Schichten rekrutiert und im Mutterhaus eine solide Ausbildung sowie eine lebenslange Berufsperspektive erhalten hatten, die ihnen an anderen Orten nicht geboten werden konnte, so wandte sich die NS-Werbung an eine Generation, der bereits breitere Bildungs- und Ausbildungswege sowie Möglichkeiten der Erwerbstätigkeit offenstanden. Dazu kam, daß die Aufstiegsmöglichkeiten innerhalb der NS-Schwesternschaft gering waren. Daß aber auch hier die "Idee des Dienens und der Pflicht" verbunden mit der bereits erwähnten politischen Dimension als Voraussetzung des Schwesternberufes angesehen wurde ${ }^{52}$, konnte die Attraktivität des neuen nationalsozialistischen Frauenberufes offensichtlich nicht erhöhen.

Seit 1938/39 versuchte die NSV verstärkt, die konfessionellen Kindergärten im Sinne ihrer Ideologie zu beeinflussen. So wurden Stoffpläne für alle Kindergärtnerinnen verbindlich, die im Fach Gesundheitsführung detaillierte Angaben über die Erziehung zur Erb- und Rassenpflege machten. Eine Kasseler Diakonisse, die bis zu diesem Zeitpunkt keine Kritik am Dritten Reich geübt hatte, erinnerte sich an ihre Reaktion, als sie von diesen Maßnahmen hörte: „Und da hab’ ich gesagt: Nun ist es aus, jetzt ist der Hitler mein Freund nicht mehr." Der eigentliche Grund für diesen Bruch lag darin, daß den Diakonissen gleichzeitig die religiöse Erziehung der Kinder untersagt wurde: „Wir sollten die Kinder zu deutschen Kindern erziehen, durften auch nicht mehr beten, von Gott etwas sagen. Und da war es ja für uns alle klar, daß wir das nicht können. Wir haben ja die Kinder betreut, um sie hinzuführen zu Jesus Christus. Da wir das ja nun nicht mehr konnten, mußten wir aus dem Kindergarten raus. " 53

Auf einem anderen Gebiet, das noch deutlicher den christlichen Auftrag verletzte, kam es innerhalb der Mutterhausdiakonie nicht zu solchen eindeutigen Reaktionen. So berichteten die Feierabendschwestern, wie sie die sog. „Kristallnacht" erlebten. Schwester Aenne aus Kassel erinnerte sich an folgende Begebenheit: „Ja, dann habe ich da noch die Kristallnacht erlebt. Es war nur eine jüdische Familie da, eine sehr nette. Ein Kind war im Kindergarten. Das wurde dann geholt und die Familie verschwand ganz. Ja dann - dann wurde es immer mehr so. "54 Schwester Berta Zimmer aus dem Diakonissenmutterhaus Königsberg erzählte:

52 So etwa Hauptamtsleiter Erich Hilgenfeldt in einem Vortrag an der Universität Berlin am 18. 2. 1939: "Ich fordere von der Schwester bedingungslosen Einsatz des Dienens und der Pflicht.“ Zit. n. Katscher, Krankenpflege 139.

53 Gespräch Heide-Marie Lauterer mit Schwester Katharina Happel, Mutterhaus Kassel (2. 5. 1986) 4.

54 Gespräch Heide-Marie Lauterer mit Schwester Aenne Linke, Mutterhaus Kassel (2.5. 1985) 2. 
"Ja, die Reichskristallnacht, die gab es. Ich bin nach Königsberg gegangen, da gab es ja eine Synagoge und dicht daneben ein Waisenhaus; das haben sie in Brand gesteckt, und die Kinder standen alle nur in Hemdchen auf der Straße. Es war ja schon November. Die Geschäfte - die Fenster eingeschlagen und alles ausgeraubt. $\mathrm{Ja}, \mathrm{ja}$, das haben wir miterlebt, aber wir konnten dagegen ja nichts machen. Später haben ja viele bereut, daß sie da nicht auch ein bißchen geholfen haben, aber es war ja immer eine Hilfe, die mit dem Tod enden konnte, oder mit Gefängnis oder $\mathrm{KZ}$, deshalb hat es niemand gewagt. " 55

In den drei Geschichten schleicht sich in das erinnernde Reden eine Distanzierung ein. Die erste Geschichte schloß mit einer Protesthandlung, aus der der Dissens mit den Maßnahmen des NS-Regimes hervorgeht. Hatte die Duldung religiöser Erziehung und Rituale bis 1938/39 noch die Zusammenarbeit von Mutterhausdiakonie und NSV ermöglicht, so war mit deren zunehmender Unterdrükkung eine Grenze erreicht, die viele Mutterhausvorstände und Diakonissen nicht überschreiten wollten. In der zweiten Geschichte liegt die Distanzierung in der positiven Charakterisierung der jüdischen Familie, doch die Schwester enthält sich eines Kommentars. In der dritten Geschichte distanziert sich die Erzählerin in dem Augenblick von dem Geschehen, als Schutzbefohlene der Mutterhausdiakonie, Kinder und Waisen, auf offener Straße mißhandelt und totgeschlagen wurden. Bei klarem Unrechtsbewußtsein rechtfertigt sie rückblickend ihr tatenloses Dabeistehen mit dem Eingeständnis ihrer und der anderen Ohnmacht zu handeln. Aber es fällt auf, daß auch diese Schwester nicht nur in ihrer Erinnerung in einer Zuschauerrolle verharrte, sondern sich als Erzählerin ebensowenig aktiv zum Inhalt ihrer Geschichte verhalten konnte. „Später“, so sagte sie, „haben ja viele bereut, daß sie da nicht auch ein bißchen geholfen haben, aber es war ja immer eine Hilfe, die mit dem Tod enden konnte." Sie selbst gehörte nicht einmal zu denen, die nachträglich Reue zeigten.

Anderen gelang es besser. So bekannte sich der Rat der EKD bereits im Oktober 1945 „im Namen der ganzen Kirche“ zur Schuld der Kirche im Dritten Reich. Der Rechtfertigung der Zuschauerrolle, dem Mangel an Zivilcourage, der Unfähigkeit, Schuld zu übernehmen, trat er in der Stuttgarter Schulderklärung entgegen: „Aber wir klagen uns an, daß wir nicht mutiger bekannt, nicht treuer gebetet, nicht fröhlicher geglaubt und nicht brennender geliebt haben. " ${ }^{56}$ In derselben Erklärung wurde davon gesprochen, daß die Bekennende Kirche „lange Jahre hindurch im Namen Jesu Christi gegen den Geist gekämpft [habe], der im nationalsozialistischen Gewaltregiment seinen furchtbaren Ausdruck gefunden hat". Nach Martin Greschats Auffassung zog der Zeitgenosse Martin Niemöller diese

55 Gespräch Heide-Marie Lauterer mit Schwester Berta Zimmer, Diakonissenmutterhaus Königsberg, heute Altenberg bei Wetzlar (28. 9. 1986).

56 Die Stuttgarter Erklärung. Der endgültige Text, in: Die Schuld der Kirche. Dokumente und Reflexionen zur Stuttgarter Schulderklärung vom 18./19. Oktober 1945, hrsg. v. Martin Greschat (München 1982) 102. 
Behauptung bereits 1945 implizit in Zweifel, wenn er gegenüber Vertretern der Ökumene der Auffassung entgegentrat, das "Schuldbekenntnis sei nicht so ganz ernst gemeint ${ }^{\text {*57. }}$.

Mit meinem Beitrag habe ich mich bemüht zu erhellen, wie dieser "Kampf“ in der Diakonie, d.h. an einer wichtigen Nahtstelle zwischen NS-Gesellschaft und Kirche sowie in einem Bereich und in einem Zeitraum, der bisher von der Forschung weitgehend vernachlässigt wurde, ausgesehen haben könnte.

57 Ebd. 98. 